\title{
Photocured Biodegradable Polymer Substrates of Varying Stiffness and Microgroove Dimensions for Promoting Nerve Cell Guidance and Differentiation
}

\author{
Lei Cai, ${ }^{\dagger}$ Li Zhang, ${ }^{\S}$ Jingyan Dong, ${ }^{\S}$ and Shanfeng Wang, ${ }^{* \dagger,+}$ \\ ${ }^{\dagger}$ Department of Materials Science and Engineering, The University of Tennessee, Knoxville, Tennessee 37996, United States \\ ${ }^{\ddagger}$ Biosciences Division, Oak Ridge National Laboratory, Oak Ridge, Tennessee 37831, United States \\ ${ }^{\S}$ Edward P. Fitts Department of Industrial and Systems Engineering, North Carolina State University, Raleigh, North Carolina 27695, \\ United States
}

\begin{abstract}
Photocross-linkable and biodegradable polymers have great promise in fabricating nerve conduits for guiding axonal growth in peripheral nerve regeneration. Here, we photocross-linked two $\operatorname{poly}(\varepsilon$-caprolactone $)$ triacrylates (PCLTAs) with number-average molecular weights of $\sim 7000$ and $\sim 10000 \mathrm{~g} \mathrm{~mol}^{-1}$ into substrates with parallel microgrooves. Cross-linked PCLTA7k was amorphous and soft, while cross-linked PCLTA10k was semicrystalline with a stiffer surface. We employed different dimensions of interests for the parallel microgrooves, that is, groove widths of $5,15,45$, and $90 \mu \mathrm{m}$ and groove depths of $0.4,1,5$, and $12 \mu \mathrm{m}$. The behaviors of rat Schwann cell precursor line (SpL201) cells

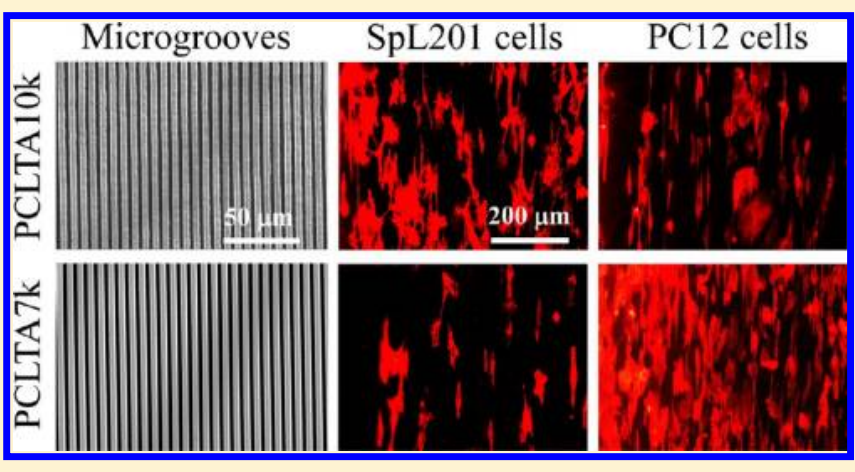
with the glial nature and pheochromocytoma (PC12) cells with the neuronal nature were studied on these microgrooved substrates, showing distinct preference to the substrates with different mechanical properties. We found different threshold sensitivities of the two nerve cell types to topographical features when their cytoskeleton and nuclei were altered by varying the groove depth and width. Almost all of the cells were aligned in the narrowest and deepest microgrooves or around the edge of microgrooves. Oriented SpL201 cell movement had a higher motility as compared to unaligned ones. After forskolin treatment, SpL201 cells demonstrated significantly upregulated S-100 and O4 on stiffer substrates or narrower microgrooves, suggesting more differentiation toward early Schwann cells (SCs). PC12 neurites were oriented with enhanced extension in narrower microgrooves. The present results not only improve our fundamental understanding on nerve cell-substrate interactions, but also offer useful conduit materials and appropriate feature dimensions to foster guidance for axonal growth in peripheral nerve regeneration.
\end{abstract}

\section{INTRODUCTION}

Peripheral nerve system (PNS) injuries with small defects can be repaired by using autografts or polymeric nerve guidance conduits for guiding axonal growth, but large-gap PNS and central nervous system (CNS) repair and regeneration are still challenging. ${ }^{1-4}$ To design optimal nerve conduits for guiding functional reconnections of neuronal tissue, researchers have investigated extensively on nerve cell-material interactions. ${ }^{1-4}$ Because nerve cells can respond to surface chemistry, topography, and stiffness, ${ }^{5-8}$ strategies of combining favorable material properties and substratum features in nerve conduits are widely used to enhance axonal growth. Despite extensive studies on microgroove guidance of nerve cells in the past, ${ }^{1,3,4}$ we used biodegradable photocross-linked polymer microgrooved substrates with distinct mechanical properties and various microgroove dimensions to demonstrate their effects on guidance and differentiation of two different nerve cell types. As compared to the cell type with the neuronal nature, a unique glial cell type that has not been studied on microgrooves was used here to show its distinct responses to both substrate stiffness and microgroove dimensions.

The advantages of using biodegradable and photocrosslinkable polymers in tissue engineering applications are their injectability and feasibility in fabricating structures with precise features via different methods, in particular, stereolithography. Nerve guides made from biodegradable materials can disappear after regeneration is fulfilled and avoid chronic nerve compression, long-term inflammatory reaction, and a secondary surgery to remove the guide. ${ }^{4}$ Many polymers of this kind have been developed, for example, poly ( $\varepsilon$-caprolactone fumarate) $(\mathrm{PCLF})^{9-12}$ and PCL acrylate (PCLA). ${ }^{13-15}$ The mechanical properties of the PCL networks made from PCLF or PCLA can be well controlled via cross-linking density or/and crystallinity in the tensile modulus range of $0.5-200 \mathrm{MPa}$, which is suitable for material handling. ${ }^{9-15}$ Among the PCL networks, the one

Received: May 6, 2012

Published: August 2, 2012 
with the highest crystallinity and stiffness was found to best support rat Schwann cell precursor line (SpL201) cell attachment, spreading, and proliferation. ${ }^{12,14}$ This PCL network was thus used to fabricate nerve conduits for guiding axonal growth. ${ }^{12}$ PCL networks tethered with poly(ethylene glycol) chains were used to make heterogeneous nerve conduits with a compositional gradient along the wall thickness to promote nerve cell functions inside the conduit while preventing undesired tissue formation on the outer wall. ${ }^{16}$ Concentricmicrogrooved substrates of cross-linked PCLTAs with various groove widths and depths prepared via replica molding were used to promote alignment and differentiation of mouse preosteoblastic MC3T3-E1 cells. ${ }^{15}$ In this study, we used parallel microgrooves instead of concentric ones and targeted a different application: nerve repair and regeneration.

During the development of nervous systems, glial cells or oriented extracellular matrix (ECM) fiber tracts allow neurons to migrate along the desired direction. ${ }^{3}$ Glial cells can form myelin to support and protect neuronal development and axon pathfinding. ${ }^{3}$ Because of the distinct functions between glial and neuronal cells, they may have different responses to the substrates with varied mechanical properties ${ }^{14,17-22}$ and topographies. ${ }^{23-26}$ As a typical glial cell type, Schwann cells (SCs) can be aligned and oriented on micropatterned substrates on silicon wafers, ${ }^{27}$ poly(dimethylsiloxane) (PDMS), ${ }^{28}$ and poly(D,L-lactide) ${ }^{29}$ to enhance neurite alignment and outgrowth. ${ }^{28,29}$ SC implants survive poorly and do not migrate when the cells fail to find axons to myelinate. ${ }^{30}$ Conditionally immortalized SpL201 cells not only retain key advantages of postnatal SCs but also can circumvent major problems associated with SCs for myelin repair. ${ }^{30}$ Pheochromocytoma (PC12) cell is an important system for studying neuronal cell communication because of its ability to extend neurites and similarity to sympathetic neurons upon treatment of nerve growth factor (NGF). ${ }^{22,31}$ PC12 cells have been reported to extend neurites with the number, length, and orientation controlled by the topographic features of micropatterned surfaces. ${ }^{31-35}$ Despite these previous reports, there still lacks a comparative study on the responses of both glial and neuronal cells to topographic features.

In this study, we have investigated the roles of both material intrinsic properties and microgroove dimensions in regulating rat SpL201 cells with the glial nature and PC12 cells with the neuronal nature. By simultaneously modulating mechanical properties and topography using the two PCLTA networks, we have correlated surface stiffness and microgroove dimensions with nerve cell attachment, proliferation, alignment, migration, and differentiation. We feel compelled to report the distinction between neuronal and glial cells in terms of responding to nonhydrogel biodegradable polymer substrates with elastic moduli greater than $1 \mathrm{MPa}$. These results could not only improve our fundamental understanding on nerve cell-material interactions but also provide guidance for fabricating nerve conduits with appropriate mechanical properties and structural features to promote nerve repair and regeneration.

\section{EXPERIMENTAL SECTION}

Materials. PCLTA was synthesized in our lab using ring-opening polymerization of $\varepsilon$-caprolactone initiated by 1,1,1-tris(hydroxymethyl) propane, followed by acrylation using acryloyl chloride in the presence of $\mathrm{K}_{2} \mathrm{CO}_{3} \cdot{ }^{14,15,36}$ PCLTA7k and 10k used in this study had number-average molecular weights $\left(M_{\mathrm{n}}\right)$ of 6700 and $9800 \mathrm{~g} / \mathrm{mol}$, and weight-average molecular weights $\left(M_{\mathrm{w}}\right)$ of 8500 and
$12300 \mathrm{~g} / \mathrm{mol}$, respectively. All other reagents used in this study were purchased from Sigma-Aldrich Co. (Milwaukee, WI) unless otherwise noted. Microfabricated silicon wafers with parallel microgrooves were prepared using standard stereolithography as templates or molds. ${ }^{15}$ To investigate nerve cell responses to the microgroove dimensions, we applied four groove widths of 5, 15, 45, and $90 \mu \mathrm{m}$ and three groove depths of 1,5 , and $12 \mu \mathrm{m}$ for each groove width. One additional groove depth of $0.4 \mu \mathrm{m}$ was also used for examining sensitivity of PC12 neurites to the grooves. The groove width was designed to be equal to the ridge width.

Preparation of Microgrooved Substrates of Cross-Linked PCLTA. The samples were photocross-linked under UV light (Spectroline, SB-100P; intensity, $4800 \mu \mathrm{w} / \mathrm{cm}^{2}$; wavelength, 315$380 \mathrm{~nm})$ with assistance of photoinitiator, phenyl bis(2,4,6-trimethyl benzoyl) phosphine oxide (BAPO; IRGACURE 819, Ciba Specialty Chemicals).${ }^{14}$ Prior to photocross-linking, two precursor solutions were prepared by dissolving $1.5 \mathrm{~g}$ of PCLTA7k or $10 \mathrm{k}$ in $500 \mu \mathrm{L}$ of $\mathrm{CH}_{2} \mathrm{Cl}_{2}$ and then mixing with $75 \mu \mathrm{L}$ of $\mathrm{BAPO} / \mathrm{CH}_{2} \mathrm{Cl}_{2}(300 \mathrm{mg} / 1.5$ $\mathrm{mL})$. The precursor solution was poured onto a precleaned microfabricated silicon wafer between two glass plates $(2 \mathrm{~mm}$, thickness) and a silicone spacer (1 mm, thickness). Homogeneous PCLTA precursor solutions filled the silicon wafers with microgrooves efficiently. The mold was then placed under UV light at a distance of $\sim 7 \mathrm{~cm}$ from the lamp head and exposed for $20 \mathrm{~min}$ to ensure sufficient cross-linking. Cross-linked PCLTA sheets with microgrooves were peeled off from the silicon mold and dried in a vacuum oven. The samples were then soaked in acetone for 2 days to remove BAPO residue and the sol fraction, followed by complete drying in vacuum. Surface patterns of the microgrooved substrates were characterized using scanning electron microscopy (SEM; S-3500, Hitachi Instruments, Tokyo, Japan) at $5 \mathrm{kV}$.

Nerve Cell Attachment, Proliferation, Distribution, and Alignment. Rat SpL201 cells were cultured in a growth medium composed of Dulbecco's modified eagle medium (DMEM; Gibco, Grand Island, NY), 10\% fetal bovine serum (FBS; Gibco), $1 \%$ penicillin/streptomycin (Gibco), and $10 \mathrm{ng} / \mathrm{mL}$ human recombinant EGF (Pepro Tech, Rocky Hill, NJ). Rat PC12 cells (ATCC, Manassas, VA) were cultured in a growth medium consisting of F-12K medium (Gibco), 15\% horse serum (Gibco), 5\% FBS, and 1\% penicillin/ streptomycin. Both cell types were cultured at $37{ }^{\circ} \mathrm{C}$ in an incubator supplied with $5 \% \mathrm{CO}_{2}$ and $95 \%$ relative humidity. The growth medium was replaced every 2-3 days, and cells were split upon $80 \%$ confluency. Prior to cell studies, all polymer substrates were sterilized in excess $70 \%$ ethanol solution for 1 day and dried completely in a vacuum. These polymer substrates were attached to the bottom of Petri dishes using autoclaved inert silicon-based vacuum grease (Dow Corning, Midland, MI) and rinsed twice with phosphate buffered saline (PBS, Gibco) before cell seeding.

SpL201 and PC12 cells were seeded on the substrates at a density of $\sim 15000$ cells $/ \mathrm{cm}^{2}$ and cultured for $4 \mathrm{~h}, 1,4$, and 7 days. At each time point, attached cells were fixed in $4 \%$ paraformaldehyde (PFA) solution for $10 \mathrm{~min}$ at room temperature. After PFA solution was removed, the cells were washed twice with PBS and permeabilized with $0.2 \%(\mathrm{v} / \mathrm{v})$ Triton X-100. The actin filaments in cells were stained with rhodamine-phalloidin (RP; Cytoskeleton, Denver, CO) for $1 \mathrm{~h}$ at $37^{\circ} \mathrm{C}$, and the nuclei were stained with $4^{\prime}, 6$-diamidino-2-phenylindole (DAPI) at room temperature. Fluorescence images were taken using an Axiovert 25 light microscope (Carl Zeiss, Germany). Cell density and shape as well as distribution and deformation of nuclei were analyzed and averaged from the fluorescence images using ImageJ software (National Institutes of Health, Bethesda, MD). Cell numbers were counted from the nuclei in at least 15 images. Cell area and length were analyzed and averaged from 20 cells at day 1 postseeding. Cell length was determined as the longer-axis length of an ellipse that approximated the cell shape. The percentage of aligned cells was analyzed over at least 100 cells. One cell was considered aligned if the angle between the direction of the microgroove and the longer axis of the cell was less than $15^{\circ}$. Nuclear circularity, defined as $4 \pi \times$ area/ perimeter ${ }^{2}$, was evaluated with a measure of 1 , indicating a perfect circle. 


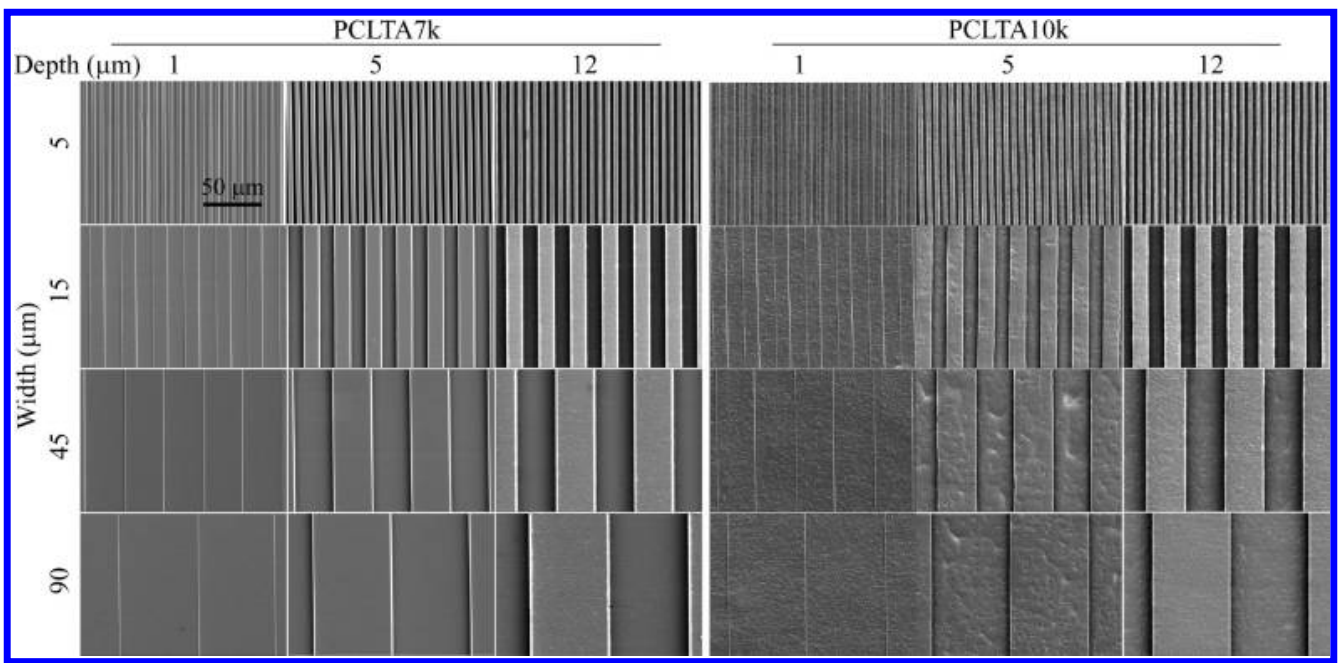

Figure 1. SEM images (top view) of microgrooved substrates of cross-linked PCLTA10k and 7k with groove widths of 5, 15, 45, and $90 \mu \mathrm{m}$ and groove depths of 1,5 , and $12 \mu \mathrm{m}$. Scale bar of $50 \mu \mathrm{m}$ is applicable to all.

SpL201 Cell Migration. SpL201 cell migration in the growth medium was conducted at the culture condition and recorded using time-lapsed fluorescence microscopy. After cells attached to the microgrooved substrates at $12 \mathrm{~h}$ postseeding, they were labeled with $0.1 \mu \mathrm{M}$ Calcein AM (Invitrogen, Carlsbad, CA) and monitored by photographing for $12 \mathrm{~h}$ at the interval of $10 \mathrm{~min}$. Cell motility was quantified by tracking at least 100 cells using ImageJ. The dislocation of the centroid of each cell was represented by a migration vector expressed as a dot in an $X Y$ diagram.

Nerve Cell Differentiation and Gene Expression. After SpL201 cells attached onto the substrates, the growth medium was replaced by the differentiation medium that was made of DMEM, 10\% FBS, $1 \%$ penicillin/streptomycin, and $20 \mu \mathrm{M}$ forskolin, instead of EGF contained in the growth medium. Upon 7-day differentiation, cells were lysed and total RNA was isolated using RNeasy Mini Kit (Qiagen, Valencia, CA). The amount of total RNA from the cells on each substrate was quantified using a Nanodrop 1000 spectrophotometer (Thermo Scientific, Wilmington, DE). Reverse transcription of isolated RNA was then performed using a DyNAmo cDNA synthesis kit (Thermo Scientific) according to the manufacturer's protocol.

The following oligonucleotide primer sequences were used: small calcium binding protein (S-100) forward, 5'-ATA GCA CCT CCG TTG GAC AG-3'; S-100 reverse, 5' -TCG TTT GCA CAG AGG ACA AG-3'; Oct-6 forward, 5'-TCC CTT TCT CTT CCC CTC TC-3'; Oct-6 reverse, 5'-GGC TCT GGT AAA ACG AAA CG-3'; protein zero (P0) forward, 5'-GGT GGT GCT GTT GCT GCT G-3', P0 reverse, 5' -TTG GTG CTT CGG CTG TGG TC-3'; p75 forward, 5' CAT CTC TGT GGA CAG CCA GA-3'; p75 reverse, $5^{\prime}$-CTC TAC CTC CTC ACG CTT GG-3'; glyceraldehyde-3-phosphate-dehydrogenase (GAPDH) forward, 5'-TCT TCA CCA CCA TGG AGA A-3'; GAPDH reverse, $5^{\prime}$-ACT GTG GTC ATG AGC CCT T-3'. Real-time PCR reactions were performed in a total volume of $25 \mu \mathrm{L}$ PCR mixture of each cDNA sample, a specific primer, and a Power SYBR Green PCR Master Mix (Applied Biosystems, Carlsbad, CA). Each PCR cycle consisted of denaturation at $94{ }^{\circ} \mathrm{C}$ for $30 \mathrm{~s}$, annealing at 55 ${ }^{\circ} \mathrm{C}$ for $30 \mathrm{~s}$, and elongation at $72{ }^{\circ} \mathrm{C}$ for $30 \mathrm{~s}$. All samples were run in triplicate using a Peltier Thermal Cycler with fluorescence detection systems (PTC-200, MJ Research). The expression level of S-100 was normalized to that of GAPDH.

To immunostain differentiated SpL201 cells, they were fixed at day 7 with $4 \%$ paraformaldehyde at room temperature for $20 \mathrm{~min}$, followed by blocking with PBS containing $0.3 \%$ Triton X-100 and $1 \%$ bovine serum albumin (BSA) at room temperature for $30 \mathrm{~min}$. Cells were stained with primary antibody of mouse monoclonal antioligodendrocyte marker O4 antibody (1:1000; R\&D Systems, Minneapolis, $\mathrm{MN}$ ) or mouse S-100 protein Ab-1 (Thermo Scientific) at $37^{\circ} \mathrm{C}$ for 1 $\mathrm{h}$ and then at $4{ }^{\circ} \mathrm{C}$ overnight. Cells were washed using PBS containing $1 \%$ BSA three times. They were then stained with secondary antibody of goat antimouse IgM-FITC (1:100; Sigma) for O4 or goat antimouse IgG-FITC (1:100; Sigma) for S-100 at $37{ }^{\circ} \mathrm{C}$ in the dark for $2 \mathrm{~h}$. After being washed with PBS three times, cells were counterstained with DAPI at room temperature for photographing. To assess O4 and S-100 protein expression in the SpL201 cells, all cells were imaged at a fixed exposure time. The expression levels of $\mathrm{O} 4$ and $\mathrm{S}$ 100 were quantified by immunofluorescence intensity using ImageJ. The average gray value of each cell was obtained from at least 20 individual cells and then normalized to the value for flat PCLTA10k substrate.

PC12 cell neurites were induced in a growth medium supplemented with $50 \mathrm{ng} / \mathrm{mL}$ NGF for 7 days. For SEM imaging, neurites were fixed in $4 \%$ PFA in $0.1 \mathrm{M}$ cacodylate solution for $10 \mathrm{~min}$ followed by a secondary fixation of $2 \%$ osmium tetroxide $\left(\mathrm{OsO}_{4}\right)$ in $0.1 \mathrm{M}$ cacodylate for $1 \mathrm{~h}$. Substrates with neurites were dehydrated through a graded ethanol series $(25 \%, 50 \%, 70 \%, 95 \%$, and $100 \%)$ and dried in vacuum. An accelerating voltage of $5 \mathrm{kV}$ was applied during imaging. PC12 cell neurites were also stained with RP. The number of neuritebearing cells, the length and angle of neurites, and the number of neurites per cell were obtained from at least 100 PC12 cells on the microgrooved substrates and compared to the flat substrates using ImageJ. Percentage of PC12 differentiation was calculated by dividing the number of neurite-bearing cells by the total number of attached cells. Neurite length was the distance from the edge of cell soma to the neurite tip. Cells with at least one neurite longer than the diameter of original, round PC12 cells (ca. $10 \mu \mathrm{m}$ ) were counted as neuritebearing cells. The number of neurites per cell was analyzed only from neurite-bearing cells. Neurite angle was quantified as the angle (always $<90^{\circ}$ ) between the direction of the neurite and its nearest microgroove edge.

Statistical Analysis. All data are presented as mean \pm standard deviation. All statistical computations were performed by analysis of variance (ANOVA) followed by Tukey post-hoc test as needed. The data were considered significantly different if the $p$-value was less than 0.05 .

\section{RESULTS}

Characterization of Microgrooved Substrates. Parallel microgrooved substrates with excellent integrity were achieved using silicon templates with well-defined surface patterns, as demonstrated in the SEM images in Figure 1. The groove depths determined from the SEM images were almost identical to the designed values, while the groove widths (ridge widths) were $4.8 \pm 0.2(4.6 \pm 0.1), 16.9 \pm 0.5(16.2 \pm 0.6), 46.0 \pm 0.5$ 
$(45.2 \pm 0.3)$, and $90.8 \pm 0.3(89.1 \pm 0.3) \mu \mathrm{m}$, respectively Crystallization-induced rougher surfaces could be seen on the substrates of semicrystalline cross-linked PCLTA10k, as compared to smoother surfaces of amorphous cross-linked PCLTA7k. They had distinct thermal and mechanical properties at $37{ }^{\circ} \mathrm{C}$ before and after cross-linking. The melting point $\left(T_{\mathrm{m}}\right)$ was 22.9 and $44.7^{\circ} \mathrm{C}$ for cross-linked PCLTA7k and 10k, respectively. ${ }^{15}$ As a result, cross-linked PCLTA7k was amorphous and soft, while PCLTA10k was semicrystalline and stiff at body temperature. ${ }^{15}$ The tensile modulus at $37{ }^{\circ} \mathrm{C}$ was $1.7 \pm 0.5 \mathrm{MPa}$ for cross-linked PCLTA7k, and it increased dramatically to $105.9 \pm 23.6 \mathrm{MPa}$ for cross-linked PCLTA10k because of enhancement from crystalline domains. ${ }^{15}$

Nerve Cell Attachment and Proliferation. SpL201 and PC12 cells seeded on the parallel microgrooves demonstrated distinct responses to both material properties and groove dimensions. Figure 2 shows actin filaments stained with RP at

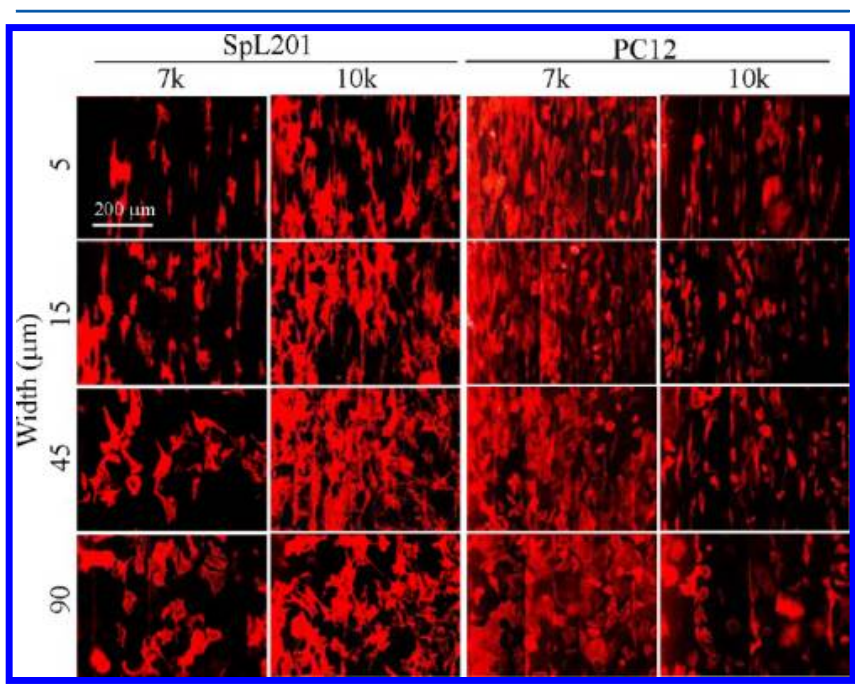

Figure 2. Fluorescence images of SpL201 (left two columns) and PC12 cells (right two columns) stained with RP at day 7 postseeding on microgrooved substrates of cross-linked PCLTA7k and 10k with a groove depth of $5 \mu \mathrm{m}$ and groove widths of $5,15,45$, and $90 \mu \mathrm{m}$. Scale bar of $200 \mu \mathrm{m}$ is applicable to all.

day 7 postseeding on microgrooves with different groove widths. SpL201 cells with the glial nature were more confluent on the stiffer substrate of cross-linked PCLTA10k than those on cross-linked PCLTA7k, consistent with our previous finding using flat substrates of cross-linked PCLDAs. ${ }^{14}$ On the contrary, PC12 cells with the neuronal nature preferred softer substrates of cross-linked PCLTA7k. When the groove width increased from 5 to $90 \mu \mathrm{m}$, cell density did not vary much, but less alignment and orientation of cytoskeleton were found for both cell types, which is elaborated in the next section. Cell numbers (Figure 3a,b) were counted and averaged from the images of cell nuclei on the substrates. In agreement with the cell images in Figure 2, cell numbers showed no statistical difference between microgrooved and flat surfaces and among different groove dimensions at all time periods for both SpL201 and PC12 cells. Unlike topological parameters, the mechanical properties of the substrates significantly affected cell attachment and proliferation for these two cell types. SpL201 cell density was always higher on stiffer cross-linked PCLTA10k regardless of surface topography, whereas PC12 cells showed an opposite trend of preferring softer substrates, which was also found on
PEGDA hydrogels with much lower moduli from 10 to 250 $\mathrm{kPa}^{17}$ The proliferation index (PI) of the cells was obtained by dividing the cell number at day 7 by the number of attached cells at $4 \mathrm{~h}$ postseeding. The PI of SpL201 cells increased from $3.30 \pm 0.29$ on cross-linked PCLTA7k to $4.31 \pm 0.34$ on crosslinked PCLTA10k. In contrast, the PI of PC12 cells decreased from $4.57 \pm 0.16$ on cross-linked PCLTA7k to $3.98 \pm 0.17$ on cross-linked PCLTA10k. Unlike the cell number, cell shape was dramatically altered by the groove width. Because of the confinement of microgrooves, smaller cell spreading area (Figure 3c,d) but larger cell length (Figure 3e,f) was found for both SpL201 and PC12 cells in the $5 \mu$ m-wide microgrooves than in the $90 \mu \mathrm{m}$-wide microgrooves and flat substrates, demonstrating significant cell elongation as a result of the "contact guidance" effect. Cross-linked PCLTA10k could elongate SpL201 cells better than cross-linked PCLTA7k for all microgrooves, whereas no statistical difference was found for PC12 cells.

Nerve Cell Alignment. Cell alignment indicates how surface topography can guide nerve cells along a desired direction. ${ }^{3}$ As shown in the fluorescence images in Figure 4a,b, cell alignment was influenced by both groove width and depth. A smaller groove width induced a higher degree of cell alignment for both SpL201 and PC12 cells. In contrast, SpL201 and PC12 cells responded differently to the groove depth. When the groove depth decreased from 12 to $1 \mu \mathrm{m}, \mathrm{SpL} 201$ cells were less aligned, but PC12 cells could still be well aligned. The percentage of aligned cells is shown in Figure $4 c, d$. More than $95 \%$ of SpL201 cells were aligned in the deepest $(12 \mu \mathrm{m})$ and narrowest $(5 \mu \mathrm{m})$ microgrooves. It sharply decreased to $\sim 40 \%$ on the microgrooves with a smaller depth of $1 \mu \mathrm{m}$. Consistently, SC alignment was reported to be reduced when the groove depth decreased from 3 to $0.5 \mu \mathrm{m} .{ }^{27}$ In contrast, PC12 cells did not exhibit a significant difference in the images when the groove depth decreased from 12 to $1 \mu \mathrm{m}$, indicating a higher threshold sensitivity than SpL201 cells. Cell alignment also decreased gradually for both cell types when the groove width increased from 5 to $90 \mu \mathrm{m}$. Furthermore, cell alignment showed time dependence for both cell types on all of the microgrooved substrates. For example, on the microgrooved substrates with the largest depth of $12 \mu \mathrm{m}$ and the smallest width of $5 \mu \mathrm{m}, 75 \pm 4 \%$ of cells were aligned along the microgrooves at $4 \mathrm{~h}$, but it increased to $99 \pm 1 \%$ at day 7 . Such time dependence of better cell alignment at longer culture time was also reported for SC alignment. ${ }^{27,28}$ Here, we did not find a significant difference in cell alignment between cross-linked PCLTA10k and $7 \mathrm{k}$ substrates with the same microgroove dimensions. On the basis of the results in Figures 2-4, we deliberately emphasized the behavior of SpL201 cells on stiffer cross-linked PCLTA10k and that of PC12 cells on softer crosslinked PCLTA7k to better present the effect of microgroove dimensions on nuclear shape and distribution, cell migration, and cell differentiation in the following discussion, whereas the comparison was performed between two cross-linked PCLTAs using flat substrates for the latter two topics.

Microgroove dimensions could also affect both shape and distribution of cell nuclei. As shown in the DAPI-stained images in Figure 5a, SpL201 cell nuclei were aligned and elongated most in the microgrooves with the largest depth and the smallest width. Cell nuclei remained unaligned on the flat substrates and $1 \mu \mathrm{m}$-deep microgrooves. The distribution of nuclei was quantified using the percentage of cell nuclei trapped in the microgrooves. As indicated in Figure 5b, $90 \%$ of nuclei 


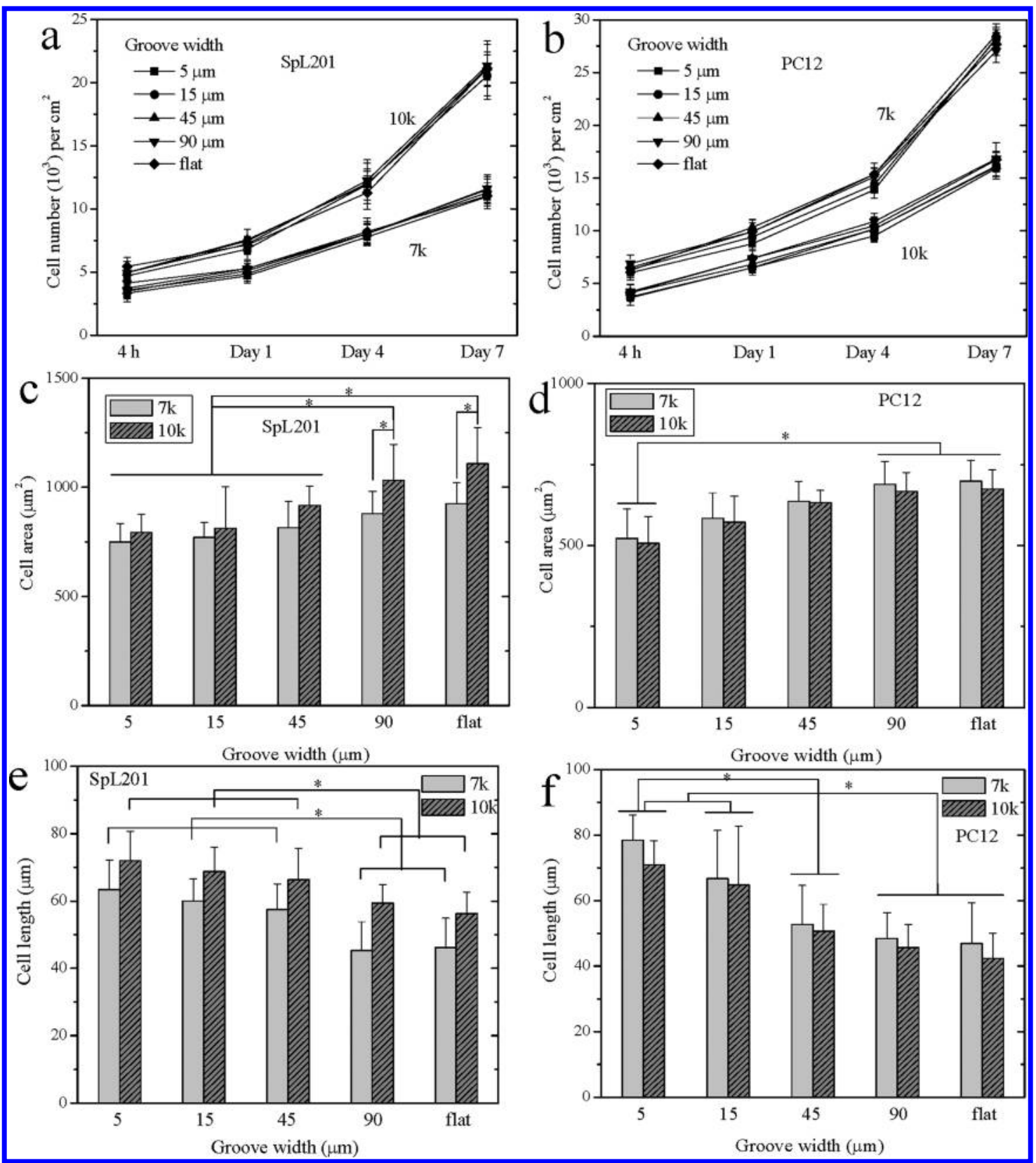

Figure 3. (a) SpL201 and (b) PC12 cell densities at 4 h, days 1, 2, and 4 postseeding, (c) SpL201 and (d) PC12 cell area at day 1, and (e) SpL201 and (f) PC12 cell length at day 1 on microgrooved substrates of cross-linked PCLTA7k and 10k with a groove depth of $5 \mu \mathrm{m}$ and groove widths of 5 , 15, 45, and $90 \mu \mathrm{m}$, as compared to flat substrates. ${ }^{*}, p<0.05 . p<0.05$ between PCLTA7k and 10k microgrooves in (e).

were confined in the microgrooves when the groove depth was $12 \mu \mathrm{m}$ and the groove width was $5 \mu \mathrm{m}$. This number decreased to $\sim 80 \%$ when the groove depth decreased to $5 \mu \mathrm{m}$. No nuclei could be confined in the microgrooves as shallow as $1 \mu \mathrm{m}$, where nuclei could ride over several microgrooves without being completely confined inside one. When the microgrooves and also the ridges were wider, fewer nuclei were trapped and aligned inside the microgrooves. Nuclei were evenly distributed on the ridges or in the microgrooves when the groove width was $90 \mu \mathrm{m}$. The percentage of cell nuclei in the microgrooves increased with culture time, resulting in better cell alignment as discussed previously. For example, it was $71 \pm 4 \%$ for day 1 but $89 \pm 6 \%$ for day 7 on the substrates with the narrowest and deepest microgrooves. It can be interpreted as that cells might descend into the microgrooves because of gravity and contact guidance, and aligned cells in the microgrooves might proliferate faster than unaligned cells on the ridges. ${ }^{3}$ Similar to cell alignment, cell nuclei were equally aligned on crosslinked PCLTA10k and $7 \mathrm{k}$ without showing the effect of substrate stiffness.

The deformation of nuclei was quantified using nuclear circularity, an indicator of the roundness of nuclei. As shown in Figure $5 c$, the most elongated nuclei with the lowest average circularity were found in the microgrooves with the highest depth of $12 \mu \mathrm{m}$ and the smallest width of $5 \mu \mathrm{m}$, which was smaller than the nuclear diameter. Cells in the microgrooves with larger widths of 45 and $90 \mu \mathrm{m}$ or a smaller depth of $1 \mu \mathrm{m}$ had nuclear circularities similar to those on the flat substrates, except for those around the microgroove edge. When the groove depth was $12 \mu \mathrm{m}$, the nuclei around the edge of the 


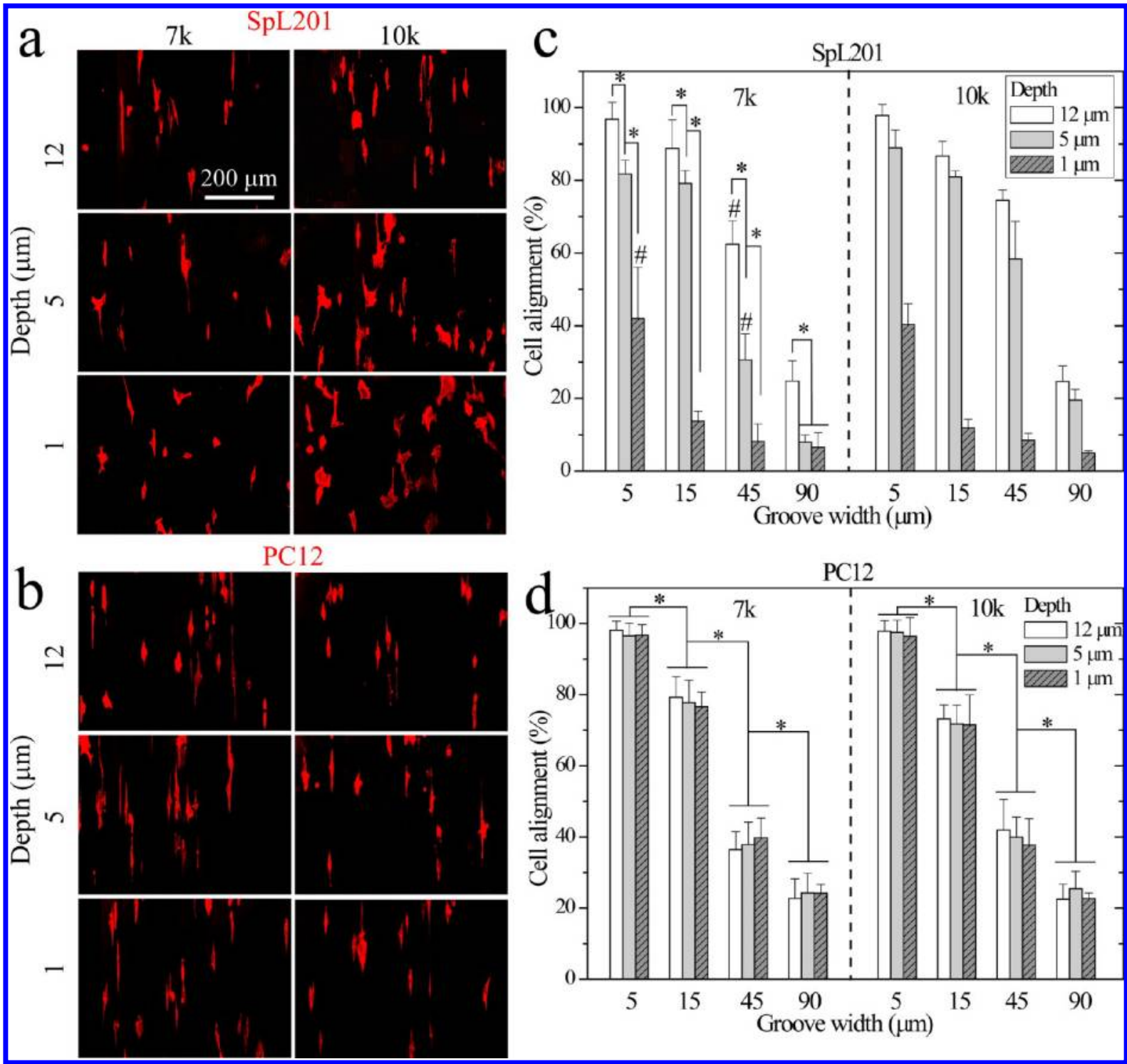

Figure 4. Fluorescence images of (a) SpL201 and (b) PC12 cells stained with RP at day 1 postseeding on microgrooved substrates of cross-linked PCLTA10k and 7k with groove depths of 1,5 , and $12 \mu \mathrm{m}$ and a groove width of $5 \mu \mathrm{m}$. Scale bar of $200 \mu \mathrm{m}$ is applicable to all. (c) SpL201 and (d) PC12 cell alignment in percentile on cross-linked PCLTA10k and 7k microgrooves with groove depths of 1,5 , and $12 \mu \mathrm{m}$ and groove widths of 5, 15,45 , and $90 \mu \mathrm{m} . *, p<0.05$. \#, $p<0.05$ relative to other groups with the same depth. $p<0.05$ between any two PCLTA10k microgrooves with the same width or depth in (c).

wider microgrooves had circularities similar to those in the narrowest microgrooves, also showing the "contact guidance" effect.

Nerve Cell Migration. Migration of glial cells is crucial in achieving connectivity and glia would pave the road first to serve as a template for the development of neurons and axon pathfinding. ${ }^{3}$ Similar to that found in nerve cell alignment, microgrooves could also influence SpL201 cell motility and migration direction, as demonstrated in Figure 6. By tracking the movement of over 100 cells in $12 \mathrm{~h}$ after cell attachment, we found a significantly higher motility for aligned cells as compared to unaligned cells. Cell motility did not vary much for different groove widths, as long as cells were aligned. Cell motility was significantly higher on flat cross-linked PCLTA10k than on flat cross-linked PCLTA7k because stiffer substrates could assist cell migration. To demonstrate the role of microgrooves in SpL201 cell migration direction, we examined cells in the microgrooves of stiffer cross-linked PCLTA10k with a depth of $5 \mu \mathrm{m}$ and a width of $45 \mu \mathrm{m}$, the size larger than the cell body. Aligned cells around the microgroove edge could persistently migrate along the direction of the microgrooves (Figure 6b). In contrast, unaligned cells migrated randomly in all directions (Figure 6c).

Nerve Cell Differentiation. Differentiation of the two nerve cell types was evaluated on two different series of crosslinked PCLTA substrates. SpL201 cells can differentiate into early SCs, which are actively involved in promoting axon growth and can be distinguished from their precursors using the expression of S-100 and the $\mathrm{O} 4$ antigen. ${ }^{30}$ Both immunostaining and gene expression were used to examine the effects of substrate stiffness and groove dimensions on the differentiation of SpL201 cells. As shown in Figure 7a, fluorescence images of differentiated SpL201 cells stained with O4 or S-100 on the stiffer substrates of cross-linked PCLTA10k clearly showed higher percentages of differentiated SpL201 cells, and the microgrooves could foster this process, in contrast to little 


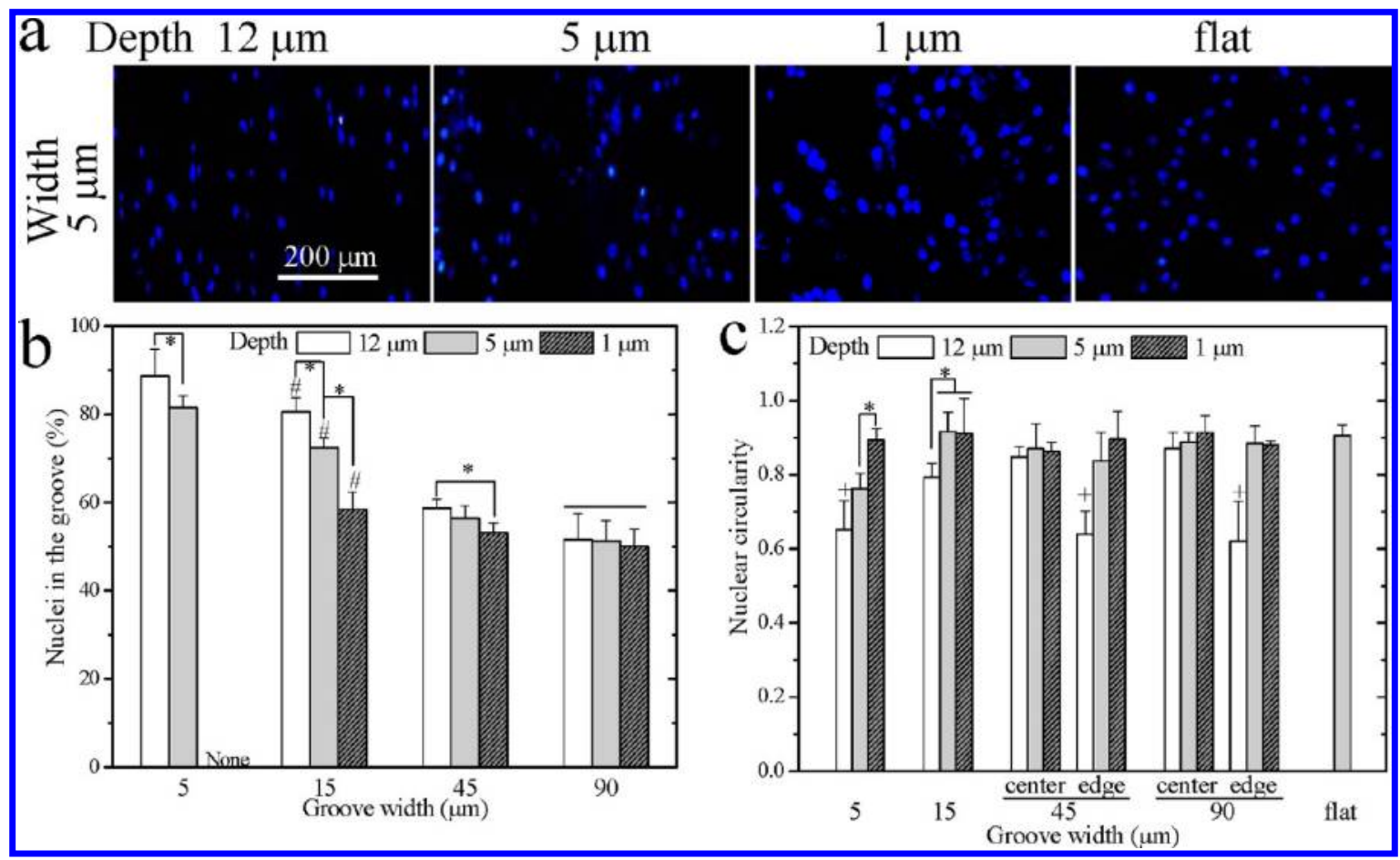

Figure 5. (a) SpL201 cell nuclei stained with DAPI at day 7 postseeding on microgrooved substrates of cross-linked PCLTA10k with groove depths of 1,5 , and $12 \mu \mathrm{m}$ and a groove width of $5 \mu \mathrm{m}$. Scale bar of $200 \mu \mathrm{m}$ is applicable to all. (b) Percentage of nuclei in the grooves and (c) nuclear circularity on microgrooved substrates with different groove dimensions. $*, p<0.05$. \#, $p<0.05$ relative to other groups with the same groove depth in (b).,$+ p<0.05$ relative to other groups in (c).

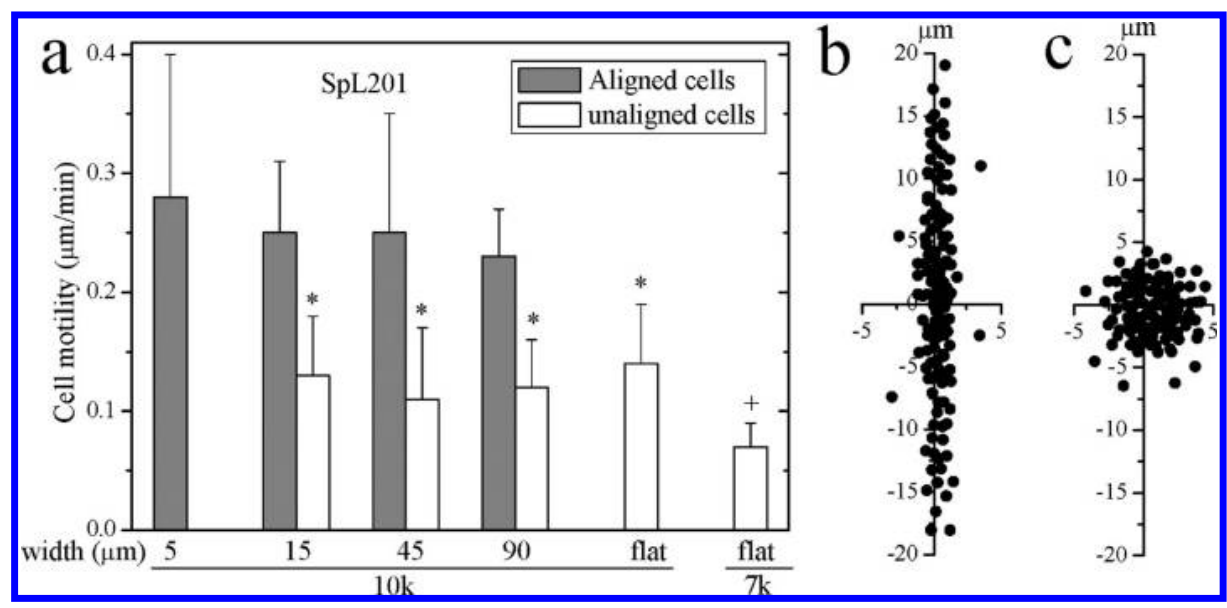

Figure 6. SpL201 cell migration on microgrooved substrates of cross-linked PCLTA10k with a groove depth of $5 \mu \mathrm{m}$ and groove widths of 5, 15, 45, and $90 \mu \mathrm{m}$, as compared to flat substrate of cross-linked PCLTA10k and 7k. (a) Cell motility of aligned cells as compared to unaligned cells. $*, p<$ 0.05 relative to unmarked groups.,$+ p<0.05$ relative to all other samples. The migration vectors of (b) aligned and (c) unaligned cells expressed as dots on microgrooved substrates of cross-linked PCLTA10k with a groove depth of $5 \mu \mathrm{m}$ and a groove width of $45 \mu \mathrm{m}$ after $30 \mathrm{~min}$.

expression in undifferentiated and differentiated SpL201 cells on flat substrates of cross-linked PCLTA7k. The immunofluorescence intensities of both markers obtained from the images are shown in Figure $7 \mathrm{~b}$. Significantly higher values were seen on microgrooved substrates with groove widths of 5-45 $\mu \mathrm{m}$ than on the flat substrate and $90 \mu \mathrm{m}$ microgrooves.

S-100 is a specific marker to distinguish early SCs from undifferentiated SpL201 cells, Oct-6 is a SC marker, and P0 is a myelin-specific gene. ${ }^{30}$ The gene expression levels of these three markers were quantified using real-time PCR, as shown in Figure 7c. The expression of S-100 was consistent with the immunostaining result in Figure 7a,b. A low level of S-100 was expressed for SpL201 cells cultured on the flat substrates of
PCLTA7k in the absence of differentiation media. S-100 expression was upregulated significantly on the flat substrates of PCLTA7k upon forskolin treatment for 7 days. Higher expression of S-100 was found on stiffer substrates of crosslinked PCLTA10k, indicating better differentiation toward a glial lineage. For SpL201 cells cultured on the microgrooved substrates, the expression level of S-100 increased dramatically when the groove width decreased, although no difference was found between the $90 \mu \mathrm{m}$-wide microgrooves and the flat substrates. In particular, the S-100 expression was upregulated by 4-fold on the microgrooved substrates with groove widths of 5 and $15 \mu \mathrm{m}$, as compared to their wider counterparts. The result indicated that SpL201 cells could differentiate into early 


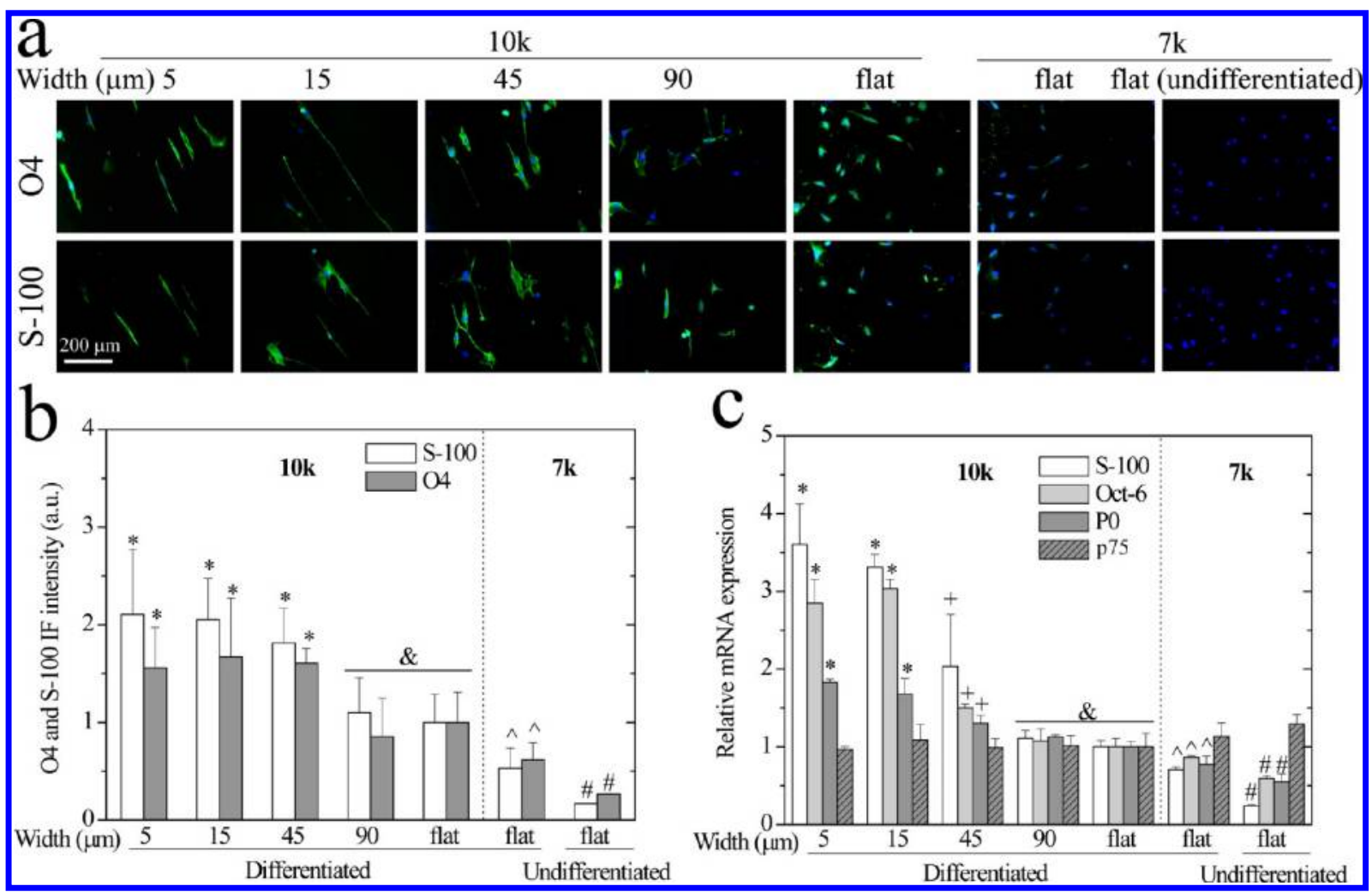

Figure 7. (a) Fluorescence images of differentiated SpL201 cells stained with O4 or S-100 (green) and DAPI (blue) on microgrooved substrates of cross-linked PCLTA10k with a groove depth of $5 \mu \mathrm{m}$ and groove widths of $5,15,45$, and $90 \mu \mathrm{m}$, flat substrates of cross-linked PCLTA10k and 7k, as compared to undifferentiated SpL201 cells on flat cross-linked PCLTA7k substrates at day 7. Scale bar of $200 \mu \mathrm{m}$ is applicable to all images. (b) Immunofluorescence (IF) intensities of O4 and S-100 normalized to those on flat cross-linked PCLTA10k substrates. $p<0.05$ between samples with different markers for each gene or antibody. (c) Relative expression levels of S-100, Oct-6, P0, and p75 gene markers to GAPDH in SpL201 cells and normalized to those on flat cross-linked PCLTA10k substrates.

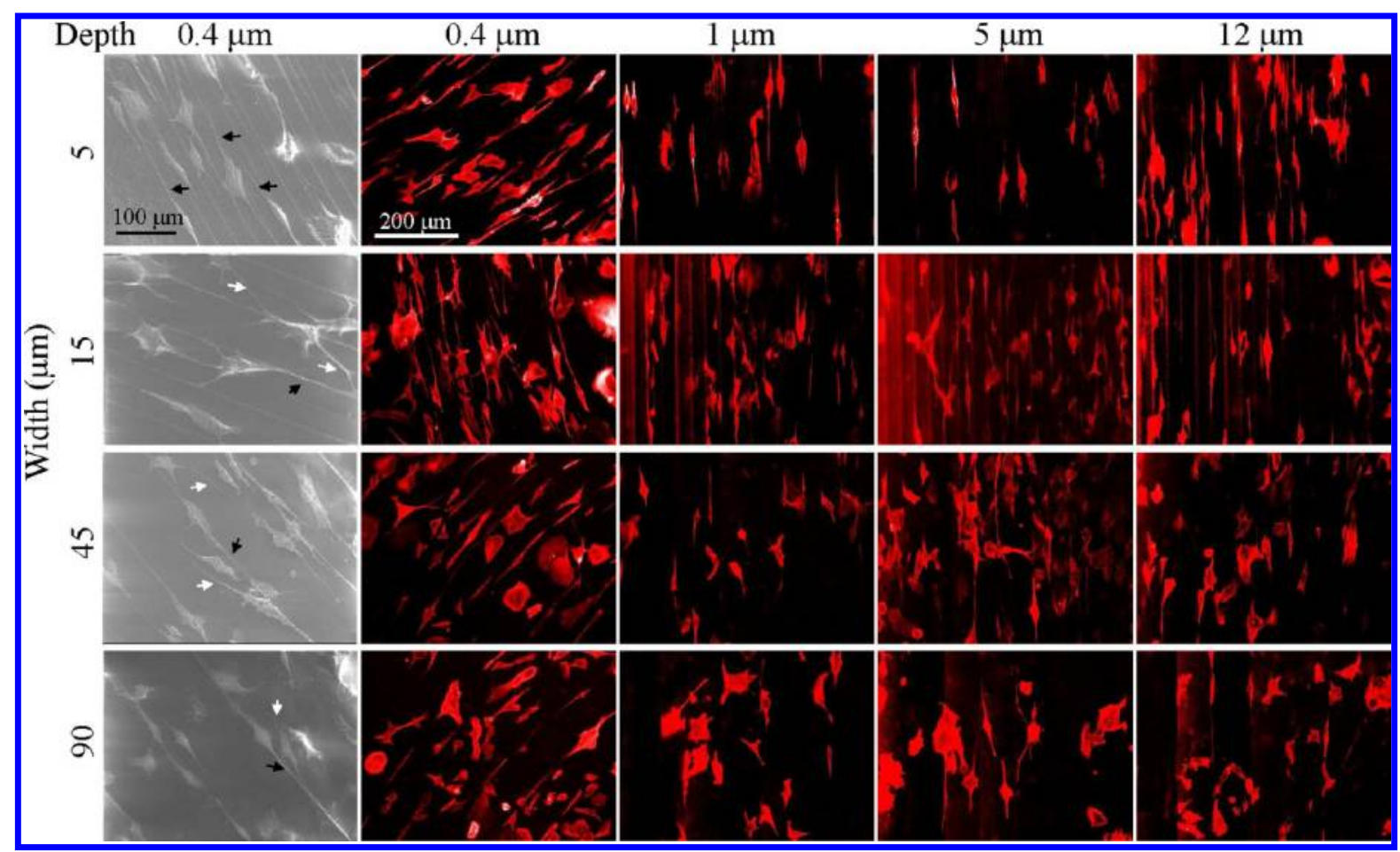

Figure 8. SEM images (first column) and fluorescence images (other columns) of PC12 cell neurite extension at day 7 postseeding on microgrooved substrates of cross-linked PCLTA7k with different groove dimensions. Black arrows indicate the neurites aligned by microgrooves. White arrows indicate the neurites not contacting the microgroove edge. Scale bar of $100 \mu \mathrm{m}$ is applicable to the first column and $200 \mu \mathrm{m}$ to others. 

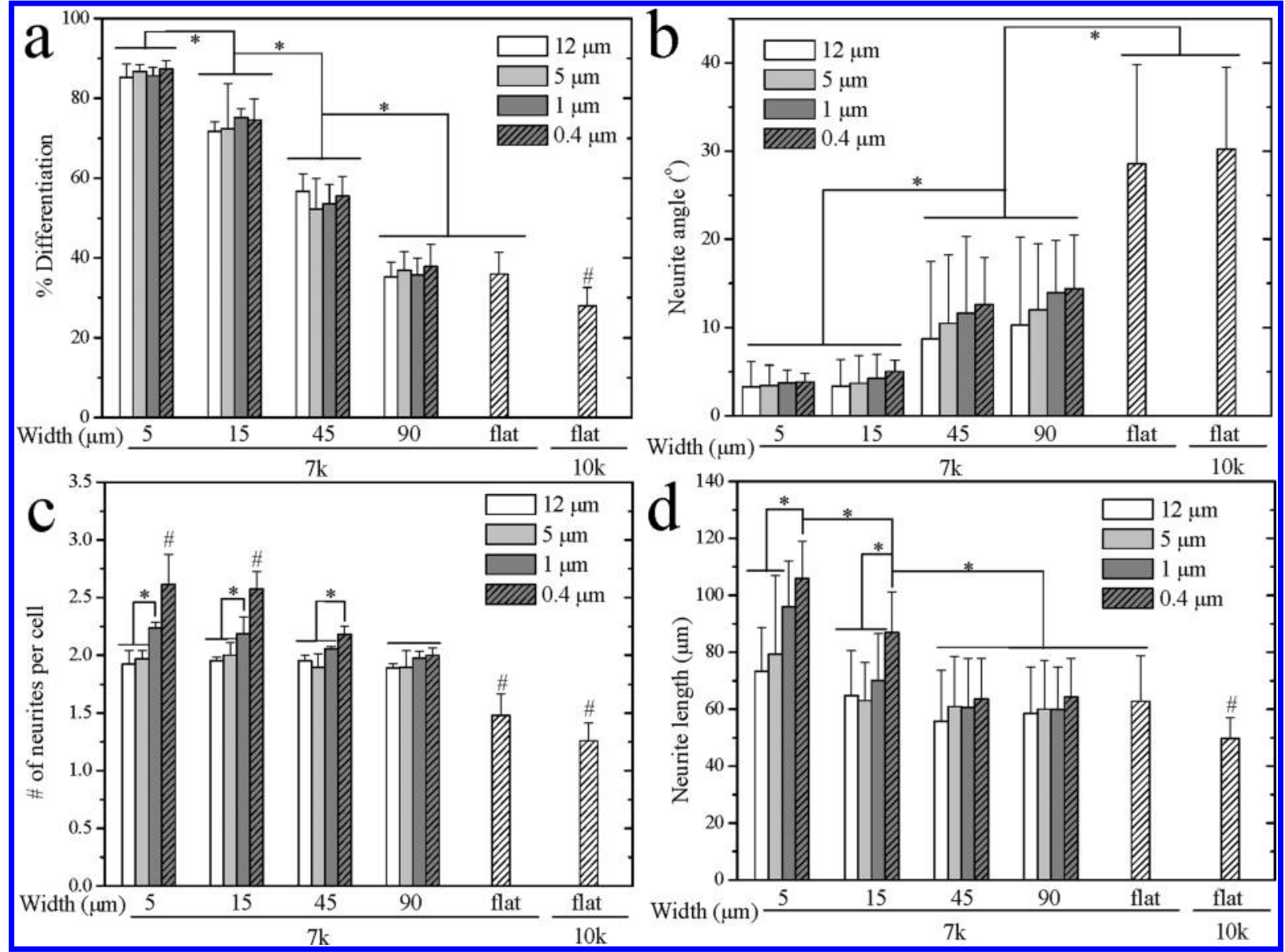

Figure 9. Quantification of PC12 cell neurites at day 7 postseeding on microgrooved substrates of cross-linked PCLTA7k with different groove dimensions, as compared to flat substrates of cross-linked PCLTA7k and 10k. (a) Percentage of differentiation, (b) neurite angle, (c) number of neurites per cell, and (d) neurite length. $*, p<0.05$. \#, $p<0.05$ relative to all other samples.

SCs better in stiffer and narrower microgrooves possibly because of more cell alignment and nuclear deformation. A previous study found that aligned SCs could have better cellcell interactions and secrete more NGF and other neurotrophic factors, ${ }^{37}$ which might benefit SC maturation. SpL201 cells were reported to upregulate Oct-6 and P0 after receiving 5-day forskolin treatment. ${ }^{30}$ Here, we quantified gene expression levels of Oct- 6 and P0 to determine the effects of substrate stiffness and groove width on the potential of SpL201 cells to differentiate into mature SCs. The trend for Oct-6 and P0 in Figure $7 \mathrm{c}$ was similar to that in S-100, showing that both higher substrate stiffness and narrower microgrooves could upregulate both markers significantly. In contrast, gene expression of $\mathrm{p} 75$, a protein expressed by neural crest-derived cells, did not vary among all of the samples possibly because this marker can be expressed by both undifferentiated and differentiated SpL201 cells. $^{30}$

PC12 cells are able to differentiate and outgrow neurites upon treatment of biochemicals such as NGF. ${ }^{32}$ As shown in the SEM and fluorescence images in Figure 8, PC12 neurites induced by NGF for 7 days could be oriented along the microgrooves when the groove width was $5 \mu \mathrm{m}$. Similar to PC12 cell proliferation, the neurites could also respond to all of the microgroove depths from 12 to $0.4 \mu \mathrm{m}$. Interestingly, when the groove width increased, much fewer neurites were found if PC12 cell soma did not contact the microgroove edge. Neurites were aligned only along the microgroove edge, as indicated by black arrows. For the neurites not contacting the microgroove edge (white arrows), they outgrew neurites in all directions without preference. Using enlarged images of differentiated PC12 cells, the neurites on microgrooved and flat substrates were quantified, as shown in Figure 9. Percentage of PC12 cell differentiation (Figure 9a) was the percentage of cells bearing neurites in the entire cell population. The groove width could strongly influence percentage of PC12 cell differentiation, while the groove depth did not. Upon 7 day NGF treatment, only $\sim 40 \%$ of PC12 cells could extend neurites on both flat substrates and the $90 \mu \mathrm{m}$-wide microgrooved ones. When the groove width decreased to $5 \mu \mathrm{m}$, the percentage of differentiation dramatically increased to $\sim 85 \%$. The angle between neurites and microgrooves (Figure $9 \mathrm{~b}$ ) was smaller on narrower microgrooves, but it was insignificantly different when the groove depth was varied. In clear contrast, the angle was much larger on flat surfaces, showing that the neurites were more randomly extended when there was no contact guidance from microgrooves.

The number of neurites per cell in Figure $9 c$ was calculated only for cells bearing neurites. Intriguingly, although all of the microgrooved substrates induced more neurites per cell as compared to the flat ones, the groove width did not affect the neurite number on the deeper microgrooves ( 5 and $12 \mu \mathrm{m}$, depth). Instead, when the microgrooves were shallower, more neurites could be induced on the narrower microgrooves. Similar to the results in the number of neurites per cell, the 
longest neurites were found on the microgrooved substrates with the smallest groove depth of $0.4 \mu \mathrm{m}$ and a groove width of $5 \mu \mathrm{m}$ (Figure 9d). SEM images in Figure 8 also showed that the neurites existed on the ridges as well as inside the microgrooves. Lower substrate stiffness could promote neurite extension by showing more cells bearing neurites, more neurites per cell, and longer neurites on cross-linked PCLTA7k than on cross-linked PCLTA10k, while the influence from microgrooves remained the same. Previous studies on PEG hydrogels showed that PC12 neurites had the greatest extension on the most flexible substrate with an elastic modulus of $\sim 30 \mathrm{kPa}^{22}$ The same trend found in our results indicated that this preference remained valid in the range of higher moduli between 1 and $100 \mathrm{MPa}$.

\section{DISCUSSION}

In this study, attachment and proliferation of both SpL201 and PC12 cells were mainly affected by substrate stiffness in the modulus range of $1-100 \mathrm{MPa}$, whereas their differentiation could be affected by microgroove dimensions, that is, groove depth and width. SpL201 cells with the glial nature attached more and proliferated faster on the stiffer substrates of crosslinked PCLTA10k. It can be explained using mechanotransduction mechanisms involving integrins, focal adhesions (FAs), and actin-myosin associations. ${ }^{3,38,39}$ A stiffer substrate can enhance integrin expression during cell adhesion and encourage maturation of FAs, which further trigger cascades of pathways to promote cell functions such as proliferation and migration. ${ }^{38,39}$ Meanwhile, actin-myosin associations can transduce mechanical signals to the nucleus, which in turn increase $\mathrm{Ca}^{2+}$ influx across the cell membrane. ${ }^{7}$ PC12 cells with the neuronal nature, however, preferred soft substrates. It might be attributed to downregulated focal adhesion kinase (FAK) expression of neurons by higher substrate stiffness. ${ }^{40}$ Previous studies have used mixed cultures of cortical neurons and astrocytes on polyacrylamide gels to show that soft gels encouraged neuronal attachment and growth but suppressed astrocyte growth, while stiff gels held an opposite trend. ${ }^{21}$ Such preference is also shown in an interesting phenomenon when an injury occurs in CNS: the stiffened tissue at the site of injury would favor glia to form a scar while inhibiting the regrowth of neurons. ${ }^{18}$

Despite no evident contribution to cell proliferation, microgrooves explored in this study could promote nerve cell alignment, guided migration, and differentiation. Both groove depth and width were important in aligning SpL201 and PC12 cells. The microgrooves with the largest depth of $12 \mu \mathrm{m}$ and the smallest width of $5 \mu \mathrm{m}$ could align almost all SpL201 cells along the microgroove direction, while fewer cells could respond to a small groove depth of $1 \mu \mathrm{m}$. The aspect ratio of the surface feature, obtained by dividing groove depth by groove width, was found to be a unified, general factor for regulating human dermal fibroblast alignment and elongation. ${ }^{41}$ Fibroblasts were better aligned and elongated when the aspect ratio was higher and the literature results were consistent, despite differences in cell types and structure variation. ${ }^{41}$ Our present results also indicated the same effect of this aspect ratio on nerve cell alignment and elongation.

Directional guidance of SCs can promote axon growth in the postinjury environment. ${ }^{28}$ After injury, SCs in the PNS would align longitudinally in columns to promote the regrowth of injured axons, leading to axonal alignment parallel to them with enhanced regeneration., ${ }^{3,28}$ Neuronal functions are also mediated by SC topography. ${ }^{28,34}$ Rat spinal neurons could be aligned to the monolayers of SCs that were oriented longitudinally in the absence of other directional cues. ${ }^{34}$ From these results on SCs, aligned SpL201 cells achieved here may have potential application in peripheral nerve repair and regeneration. Moreover, directional migration with much higher motility was found for microgroove-aligned SpL201 cells, as compared to unaligned cells. This result suggested that SpL201 cells could migrate along the preset direction with a higher velocity to achieve quicker path pavement for neurons and better capability for bridging injured nerve gaps if microgrooves with appropriate dimensions were introduced inside polymer nerve conduits. Similarly, fibroblast and epithelial cells with a more elongated shape were also reported to migrate faster along the microgrooves than cells with a nearly round shape, while their transverse movement across microgrooves was inhibited. ${ }^{42,43}$ Actin filaments in cells were also reorganized and aligned along the underlying microgrooves during migration. ${ }^{43}$

Microgrooved surfaces could align the cytoskeleton of SpL201 cells and deform the nuclei when the groove width (e.g., $5 \mu \mathrm{m}$ in this study) was smaller than the nuclei. S-100 gene expression could be significantly upregulated when SpL201 cells were cultured on microgrooved substrates with narrow grooves in differentiation media. One explanation is that gene expression could be strongly influenced by microgrooveinduced cell alignment, cytoskeletal organization, and nuclear deformation. ${ }^{23,44}$ A study showed that microgrooves can induce differentiation of human mesenchymal stem cells into neuronal lineage through alignment. ${ }^{45}$ Nuclear deformation may lead to changes in the positions of chromosomes that are related to specific gene expression and protein synthesis. ${ }^{37,46}$ Meanwhile, SpL201 cells confined in the microgrooves had higher motilities, better cell-cell contact, and higher concentrations of ECM proteins and regulatory factors, which might all assist cell maturation. The upregulation of S-100 and $\mathrm{O} 4$ induced by the microgrooves is an indicator for early SCs, which can generate myelin in coculture with neurons to ensheath and associate with axons in vitro. ${ }^{30}$ Differentiated SpL201 cells can also remyelinate denuded axons after transplantation in an adult rat spinal cord. ${ }^{30}$ As compared to featureless nerve conduits, conduits with microgrooves on the inner wall have been demonstrated to align SCs and enhance peripheral nerve regeneration. ${ }^{39}$

Microgrooved substrates with depths of $0.4-12 \mu \mathrm{m}$ all could orient and promote PC12 cell neurite extension upon NGF treatment, indicating a much higher sensitivity of PC12 cells toward groove features than SpL201 cells. As compared to the groove depth, the groove width demonstrated a more determining role in the percentage of cells bearing neurites and orientation of neurites. As the groove width decreased from 90 to $5 \mu \mathrm{m}$, significantly more cells could extend neurites, and the neurites were more parallel to the microgrooves. The mechanism for this phenomenon may be that filopodia and lamellipodia at the leading edge of neurites can first explore the surrounding surface topography and the traction forces are generated in contacts to push and pull neurites forward via microtubules and actin filaments. ${ }^{47}$ When the groove width is smaller, filopodia tend to contact the microgroove edge and exert a greater traction force to advance neurites along the microgrooves. ${ }^{3,33,47}$ In this study, the smallest groove depth of $0.4 \mu \mathrm{m}$ could also increase the number of neurites per cell and the neurite length. Because microtubules and actin filaments are inflexible, neurites are constrained and can only extend in one 
or two directions along the microgrooves with reduced branches when the groove depth is high. ${ }^{3,32,33}$ These reports are in good agreement with our findings that the narrowest and shallowest microgrooves had most neurites per cell and longest neurites. When the groove depth is smaller, neurites are able to stay inside the microgrooves and also on the ridge, leading to less confinement on neurites, which can promote their outgrowth because physical space is important for neurites to be protruded from the soma, ${ }^{32,33}$ and more cell-cell communications with adjacent cell soma, which may benefit neurite growth. ${ }^{3}$ Similarly, PC12 cells cultured in microchannels were reported to extend neurites twice longer than those on flat surfaces. ${ }^{33}$ Nevertheless, the underlying mechanism on the effect of groove depth merits further studies.

Besides improving our understanding on nerve cellbiomaterial interactions, this study also provides biodegradable substrates with suitable physicochemical characteristics and structural features for nerve regeneration. In future, remyelination in coculturing SpL201 cells with dorsal root ganglion neurons will be performed on these microgrooved surfaces and conduits. On the basis of the present results, we suggest that semicrystalline PCLTA networks with narrow microgrooves is an excellent nerve conduit candidate material because it can promote SpL201 cell proliferation, migration, and maturation to facilitate the development of neurites, which actively interact with the soft and patterned SpL201 cells underneath instead of with the stiff conduit wall. Nerve guidance conduits will also be fabricated with microgrooves on the inner wall and will be tested both in vitro and in vivo for the repair of PNS and CNS.

\section{CONCLUSIONS}

Parallel microgrooves with well-defined groove dimensions (depth and width) and distinct mechanical properties have been fabricated using two biodegradable and photocrosslinkable PCLTAs with different molecular weights. Both intrinsic material properties and feature dimensions influenced SpL201 and PC12 cell behavior significantly. Cell proliferation was better supported on stiffer substrates for SpL201 cells with the glial nature but on softer substrates for PC12 cells with the neuronal nature. Cell alignment and nuclear deformation were found to be significantly altered on narrower and deeper microgrooves. SpL201 cell motility along the microgroove direction was promoted when the cells were aligned. Furthermore, narrower microgrooves could help SpL201 differentiate into early SCs more quickly, indicated by the upregulation of S-100 and O4. PC12 neurites were oriented as well with significantly better extension on narrower but shallower microgrooves. These surface features can be potentially fabricated on the inner wall of polymer nerve conduits to provide guidance for cell regenerative functions and axon growth in peripheral nerve repair and regeneration.

\section{AUTHOR INFORMATION}

\section{Corresponding Author}

*Tel.: (865) 974-7809. Fax: (865) 974-4115. E-mail: swang16@utk.edu.

\section{Notes}

The authors declare no competing financial interest.

\section{ACKNOWLEDGMENTS}

This work was supported by the start-up fund and professional development award from the University of Tennessee and
National Science Foundation (DMR-11-06142, to S.W.). Rat SpL201 cells were a kind gift from Dr. Anthony J. Windebank's laboratory at Mayo Clinic. Fabrication of silicon molds was performed in Dr. Dong's laboratory with the help of the startup fund from North Carolina State University.

\section{REFERENCES}

(1) Schmidt, C. E.; Leach, J. B. Neural tissue engineering: strategies for repair and regeneration. Annu. Rev. Biomed. Eng. 2003, 5, 293.

(2) Madigan, N. N.; McMahon, S.; O’Brien, T.; Yaszemski, M. J.; Windebank, A. J. Current tissue engineering and novel therapeutic approaches to axonal regeneration following spinal cord injury using polymer scaffolds. Respir. Physiol. Neurobiol. 2009, 169, 183.

(3) Hoffman-Kim, D.; Mitchel, J. A.; Bellamkonda, R. V. Topography, cell response, and nerve regeneration. Annu. Rev. Biomed. Eng. 2010, 2, 203.

(4) Wang, S.; Cai, L. Polymers for fabricating nerve conduits. Int. J. Polym. Sci. 2010, 138686.

(5) Saltzman, W. M.; Kyriakides, T. R. Cell interactions with polymers. In Principles of Tissue Engineering, 3rd ed.; Lanza, R., Langer, R, Vacanti, J., Eds.; Elsevier Academic Press: San Diego, CA, 2007; pp 279-296.

(6) Harbers, G. M.; Grainger, D. W. Cell-material interactions: fundamental design issues for tissue engineering and clinical considerations. In Introduction to Biomaterials; Guelcher, S. A., Hollinger, J. O., Eds.; CRC Press: Boca Raton, FL, 2005; pp 15-45.

(7) Discher, D. E.; Janmey, P.; Wang, Y.-L. Tissue cells feel and respond to the stiffness of their substrate. Science 2005, 310, 1139.

(8) Yu, L. M. Y.; Leipzig, N. D.; Shoichet, M. S. Promoting neuronal adhesion and growth. Mater. Todav 2008, 11, 36.

(9) Wang, S.; Kempen, D. H.; Simha, N. K.; Lewis, J. A.; Windebank, A. J.; Yaszemski, M. J.; Lu, L. Photo-crosslinked hybrid polymer networks consisting of poly(propylene fumarate) and poly(caprolactone fumarate): controlled physical properties and regulated bone and nerve cell responses. Biomacromolecules 2008, 9, 1229.

(10) Wang, S.; Lu, L.; Gruetzmacher, J. A.; Currier, B. L.; Yaszemski, M. J. Synthesis and characterizations of biodegradable and photocrosslinkable poly( $\varepsilon$-caprolactone fumarate), poly(ethylene glycol fumarate) and their amphiphilic copolymer. Biomaterials 2006, 27, 832.

(11) Wang, S.; Yaszemski, M. J.; Gruetzmacher, J. A.; Lu, L. Photocrosslinked poly( $\varepsilon$-caprolactone fumarate) networks: roles of crystallinity and crosslinking density in determining mechanical properties. Polvmer 2008, 49, 5692.

(12) Wang, S.; Yaszemski, M. J.; Knight, A. M.; Gruetzmacher, J. A.; Windebank, A. J.; Lu, L. Physical properties and biological evaluations of photo-crosslinked poly ( $\varepsilon$-caprolactone fumarate) networks for peripheral nerve regeneration. Acta Biomater. 2009, 5, 1531.

(13) Cai, L.; Wang, S. Parabolic dependence of material properties and cell behavior on the composition of polymer networks via simultaneously controlling crosslinking density and crystallinity. Biomaterials 2010, 31, 7423.

(14) Cai, L.; Wang, S. Poly ( $\varepsilon$-caprolactone) acrylates synthesized using a facile method for fabricating networks to achieve controllable physicochemical properties and tunable cell responses. Polvmer 2010, 51, 164 .

(15) Wang, K.; Cai, L.; Zhang, L.; Dong, J.; Wang, S. Biodegradable photo-crosslinked polymer substrates with concentric microgrooves for regulating MC3T3-E1 cell behavior. Adv. Healthcare Mater. 2012, $1,292$.

(16) Cai, L.; Lu, J.; Sheen, V.; Wang, S. Lubricated biodegradable polymer networks for regulating nerve cell behavior and fabricating nerve conduits with a compositional gradient. Biomacromolecules 2012, 13, 358 .

(17) Cai, L.; Lu, J.; Sheen, V.; Wang, S. Promoting nerve cell functions on hydrogels grafted with poly(L-lysine). Biomacromolecules 2012, 13, 342. 
(18) Georges, P. C.; Janmey, P. A. Cell type-specific response to growth on soft materials. L. Appl. Phvsiol. 2005, 98, 1547.

(19) Nemir, S.; West, J. L. Synthetic materials in the study of cell response to substrate rigidity. Ann. Biomed. Eng. 2009, 38, 2.

(20) Flanagan, L. A.; Ju, Y. E.; Marg, B.; Osterfield, M.; Janmey, P. A. Neurite branching on deformable substrates. Neuroreport 2002, 13, 2411

(21) Georges, P. C.; Miller, W. J.; Meaney, D. F.; Sawyer, E. S.; Janmey, P. A. Matrices with compliance comparable to that of brain tissue select neuronal over glial growth in mixed cortical cultures. Biophvs. I. 2006, 90, 3012.

(22) Gunn, J. W.; Turner, S. D.; Mann, B. K. Adhesive and mechanical properties of hydrogels influence neurite extension. $I$. Biomed. Mater. Res. 2005, 72A, 91.

(23) Bettinger, C. J.; Langer, R.; Borenstein, J. T. Engineering substrate topography at the micro- and nanoscale to control cell function. Angew. Chem. Int. Ed. 2009, 48, 5406.

(24) Khan, S.; Newaz, G. A comprehensive review of surface modification for neural cell adhesion and patterning. L. Biomed. Mater. Res. 2010, 93A, 1209.

(25) Curtis, A.; Wilkinson, C. Topographical control of cells. Biomaterials 1997, 18, 1573.

(26) Lim, J. Y.; Donahue, H. J. Cell sensing and response to microand nanostructured surfaces produced by chemical and topographic patterning. Tissue Eng. 2007, 13, 1879.

(27) Hsu, S.-H.; Chen, C.-Y.; Lu, P. S.; Lai, C.-S.; Chen, C.-J. Oriented Schwann cell growth on microgrooved surfaces. Biotechnol. Bioeng. 2005, 92, 579.

(28) Bruder, J. M.; Lee, A. P.; Hoffman-Kim, D. Biomimetic materials replicating Schwann cell topography enhance neuronal adhesion and neurite alignment in vitro. I. Biomater. Sci., Polvm. Ed. 2007, 18, 967.

(29) Miller, C.; Jeftinija, S.; Mallapragada, S. Micropatterned Schwann cell-seeded biodegradable polymer substrates significantly enhance neurite alignment and outgrowth. Tissue Eng. 2001, 7, 705.

(30) Lobsiger, C. S.; Smith, P. M.; Buchstaller, J.; Schweitzer, B.; Franklin, R. J.; Suter, U.; Taylor, V. SpL201: A conditionally immortalized Schwann cell precursor line that generates myelin. Glia 2001, 36, 31.

(31) Foley, J. D.; Grunwald, E. W.; Nealey, P. F.; Murphy, C. J. Cooperative modulation of neuritogenesis by $\mathrm{PC} 12$ cells by topography and nerve growth factor. Biomaterials 2005, 26, 3639.

(32) Yao, L.; Wang, S.; Cui, W.; Sherlock, R.; O’Connell, C.; Damodaran, G.; Gorman, A.; Windebank, A.; Pandit, A. Effect of functionalized micropatterned PLGA on guided neurite growth. Acta Biomater. 2009, 5, 580.

(33) Mahoney, M. J.; Chen, R. R.; Tan, J.; Saltzman, W. M. The influence of microchannels on neurite growth and architecture. Biomaterials 2005, 26, 771.

(34) Thompson, D. M.; Buettner, H. M. Neurite outgrowth is directed by Schwann cell alignment in the absence of other guidance cues. Ann. Biomed. Eng. 2006, 34, 161.

(35) Houchin-Ray, T.; Swift, L. A.; Jang, J.-H.; Shea, L. D. Patterned PLG substrates for localized DNA delivery and directed neurite extension. Biomaterials 2007, 28, 2603.

(36) Cai, L.; Wang, S. Elucidating colorization in the functionalization of hydroxyl-containing polymers using unsaturated anhydrides/ acyl chlorides in the presence of triethylamine. Biomacromolecules 2010, 11, 304

(37) Hsu, S.-H.; Lu, P. S.; Ni, H.-C.; Su, C.-H. Fabrication and evaluation of microgrooved polymers as peripheral nerve conduits. Biomed. Microdevices 2007, 9, 665.

(38) Chen, C. S. Mechanotransduction - a field pulling together? L. Cell Sci. 2008, 121, 3285.

(39) Geiger, B.; Spatz, J. P.; Bershadsky, A. D. Environmental sensing through focal adhesions. Nat. Rev. Mol. Cell Biol. 2009, 10, 21.

(40) Jiang, F. X.; Yurke, B.; Firestein, B. L.; Langrana, N. A. Neurite outgrowth on a DNA crosslinked hydrogel with tunable stiffnesses. Ann. Biomed. Eng. 2008, 36, 1565.
(41) Crouch, A. S.; Miller, D.; Luebke, K. J.; Hu, W. Correlation of anisotropic cell behaviors with topographic aspect ratio. Biomaterials 2009, 30, 1560.

(42) Kaiser, J.-P.; Reinmann, A.; Bruinink, A. The effect of topographic characteristics on cell migration velocity. Biomaterials 2006, 27, 5230.

(43) Dalton, B. A.; Walboomers, X. F.; Dziegielewski, M.; Evans, M. D. M.; Taylor, S.; Jansen, J. A.; Steele, J. G. Modulation of epithelial tissue and cell migration by microgrooves. I. Biomed. Mater. Res. 2001, 56, 195.

(44) Dah, K. N.; Ribeiro, A. J. S.; Lammerding, J. Nuclear shape, mechanics, and mechanotransduction. Circ. Res. 2008, 102, 1307.

(45) Thomas, C. H.; Collier, J. H.; Sfeir, C. S.; Healy, K. E. Engineering gene expression and protein synthesis by modulation of nuclear shape. Proc. Natl. Acad. Sci. U.S.A. 2002, 99, 1972.

(46) Yim, E. K. F.; Pang, S. W.; Leong, K. W. Synthetic nanostructures inducing differentiation of human mesenchymal stem cells into neuronal lineage. Exp. Cell Res. 2007, 313, 1820.

(47) Alberts, B.; Bray, D.; Lewis, J.; Raff, M.; Roberts, K.; Watson, J. Molecular and Cellular Biology of the Cell; Garland Publishing: New York, NY, 1994. 